\title{
Near complete response to Pembrolizumab in microsatellite-stable metastatic sebaceous carcinoma
}

\author{
Evidio Domingo-Musibay ${ }^{1,6^{*}}$ D, Paari Murugan ${ }^{2,6}$, Alessio Giubellino ${ }^{2,6}$, Sandeep Sharma ${ }^{3}$, Daniel Steinberger ${ }^{3}$,
} Jianling Yuan ${ }^{4,6}$, Matthew A. Hunt ${ }^{5,6}$, Emil Lou ${ }^{1,6}$ and Jeffrey S. Miller ${ }^{1,6}$

\begin{abstract}
Background: Sebaceous carcinoma is an aggressive adnexal skin tumor with a predilection for the eyelids and sebaceous glands of the head and neck.

Case presentation: A 73 year-old man presented with confusion and was found to have widely disseminated sebaceous carcinoma with metastases to brain, lungs, liver, bowel, lymph nodes, and bone. Following initial treatment of the brain metastases with surgery he received post-operative radiosurgery. He then began systemic immunotherapy with pembrolizumab. After 6 months, he developed a near complete response to therapy by irRECIST and RECIST v.1.1. The response was associated with circulating CD8+ T cells with central memory (CM) and effector memory (EM) phenotype and mature CD16 + CD57+ NK cells. During treatment the patient developed adrenal insufficiency requiring high-dose systemic corticosteroids and later adrenal replacement therapy. After 12-months of follow-up he showed imaging evidence of progression in liver, mediastinum, and abdominal lymph nodes. Given persistent, strong PD-L1 expression he resumed pembrolizumab therapy and showed radiographic evidence of an ongoing response to therapy.

Conclusions: This is the first report describing objective clinical and radiographic responses following immunotherapy for widely metastatic sebaceous carcinoma. The dramatic therapeutic response to pembrolizumab was associated with peripheral blood circulating memory T cells and mature Natural Killer cells after 6 months (24 weeks) of therapy. This report supports prospective clinical trials of anti-PD1 checkpoint blockade for metastatic sebaceous carcinoma.
\end{abstract}

Keywords: Sebaceous carcinoma, Pembrolizumab, Immunotherapy, Anti-PD1, Skin cancer5

\section{Background}

Sebaceous carcinoma (SC) is an uncommon form of skin adnexal tumor, often arising from sebaceous glands of the eyelid [1], and head and neck [2]. Periocular primary tumors comprise more than half of cases [3], and sebaceous carcinoma (SC) is the third most common eyelid tumor, after basal cell and squamous cell carcinoma $[1,4]$. The overall incidence is rising [4], but cases are estimated to be 1 to 2 per 1,000,000 in the US, based on a recent review

\footnotetext{
* Correspondence: musib024@umn.edu

${ }^{1}$ Department of Medicine, Division of Hematology, Oncology and

Transplantation, University of Minnesota, 420 Delaware Street SE, MMC480, Minneapolis, MN 55455, USA

${ }^{6}$ Masonic Cancer Center, University of Minnesota, Minneapolis, MN 55455, USA

Full list of author information is available at the end of the article
}

of the Surveillance Epidemiology and End Results (SEER) database [5].

Optimal treatment of metastatic sebaceous malignancy has not been firmly established. To date, treatment approaches have been adapted from regimens used to treat head and neck cancers, with several retrospective series showing effectiveness of multi-agent cisplatin-based chemotherapy $[6,7]$. While the sporadic form of SC is not generally associated with mutations in DNA mismatch repair genes, cases associated with Muir-Torre and microsatellite instability (MSI) are likely to respond to immunotherapy $[8,9]$. Anti-PD1 checkpoint inhibitors are approved for malignant melanoma $[10,11]$ and merkel cell carcinoma, a polyomavirus associated skin adnexal tumor $[12,13]$. To date, there have been no clinical trials or case

(c) The Author(s). 2018 Open Access This article is distributed under the terms of the Creative Commons Attribution 4.0 International License (http://creativecommons.org/licenses/by/4.0/), which permits unrestricted use, distribution, and 
reports describing successful use of immunotherapy for sebaceous carcinoma tumors.

\section{Case presentation}

Herein, we describe the case of a 73 year-old man in good health, who developed widely disseminated sebaceous carcinoma including metastases to brain, visceral organs, lymph nodes, and bone.

He initially presented in late October 2016 for removal of a rapidly growing nodule in the anterior abdominal wall. Two days later he developed confusion, urinary incontinence and progressive aphasia. Emergent magnetic resonance imaging (MRI) of the brain showed 4 enhancing gray-white matter junctional lesions, the two largest measured $3.8 \times 3.3 \mathrm{~cm}$ in the right frontal lobe and $2.3 \times 2.5 \mathrm{~cm}$ in the left frontal lobe. Two smaller enhancing lesions in the right parietal lobe measured $8 \mathrm{~mm}$ and $4 \mathrm{~mm}$ in diameter. In November 2016, he underwent craniotomy and resection of bilateral frontal lobe tumors, and he made a full neurologic recovery and went on to receive post-operative gamma knife radiosurgery to the resection cavities and the small parietal brain lesions (Fig. 1).

His case was reviewed in melanoma tumor boards at the Masonic Cancer Clinic, University of Minnesota. Sections of tumor revealed sheets of epithelial cells with moderate eosinophilic cytoplasm and areas of tumor infiltrating lymphocytes (Fig. 2a). Cells exhibited nuclear pleomorphism and increased mitotic activity (Fig. 2b), desmoplastic stromal reaction and necrosis (Fig. 2c). Immunohistochemical staining was positive for cytokeratin AE1/AE3 and cytokeratin 7, and negative for S100, HMB45, Melan-A, CD45, calretinin, ERG, p40, TTF1, CDX2, and GATA3. The immunoprofile ruled out melanoma, mesothelioma, lymphoma, sarcoma with epithelioid features, and most visceral carcinomas. Microscopic examination revealed intracytoplasmic lipid vesicles (Fig. 2d), confirmed by diffuse membranous reactivity for adipophilin $[14,15]$ (Fig. 2f and g). The findings supported a histopathologic diagnosis of sebaceous carcinoma. Importantly, additional tumor testing confirmed high expression of PD-L1 in 100\% of tumor cells (Fig. 2h). Commercial genomic testing using next-generation sequencing (Foundation Medicine, Massachusetts, USA) confirmed the tumor was microsatellite stable and carried a mutational burden of 17 mutations/Mb. Table 1 also shows various somatic mutations in genes for regulatory transcription factors, DNA repair proteins, growth factor receptors, and targetable MAPK signaling proteins. Several of the affected genes have also been described in cases of sebaceous carcinoma reported in the COSMIC (cancer.sanger.ac.uk) database [16].

Initial staging positron emission tomography-computed tomography (PET/CT) revealed evidence of widely disseminated disease involving lung and liver, muscle, bone, and multi-compartment bulky lymphadenopathy in chest and abdomen (Fig. 3a). Standard chemotherapy approaches using platinum-based chemotherapy were reviewed. However, the patient and family strongly favored a less toxic therapy, considering advanced age and quality

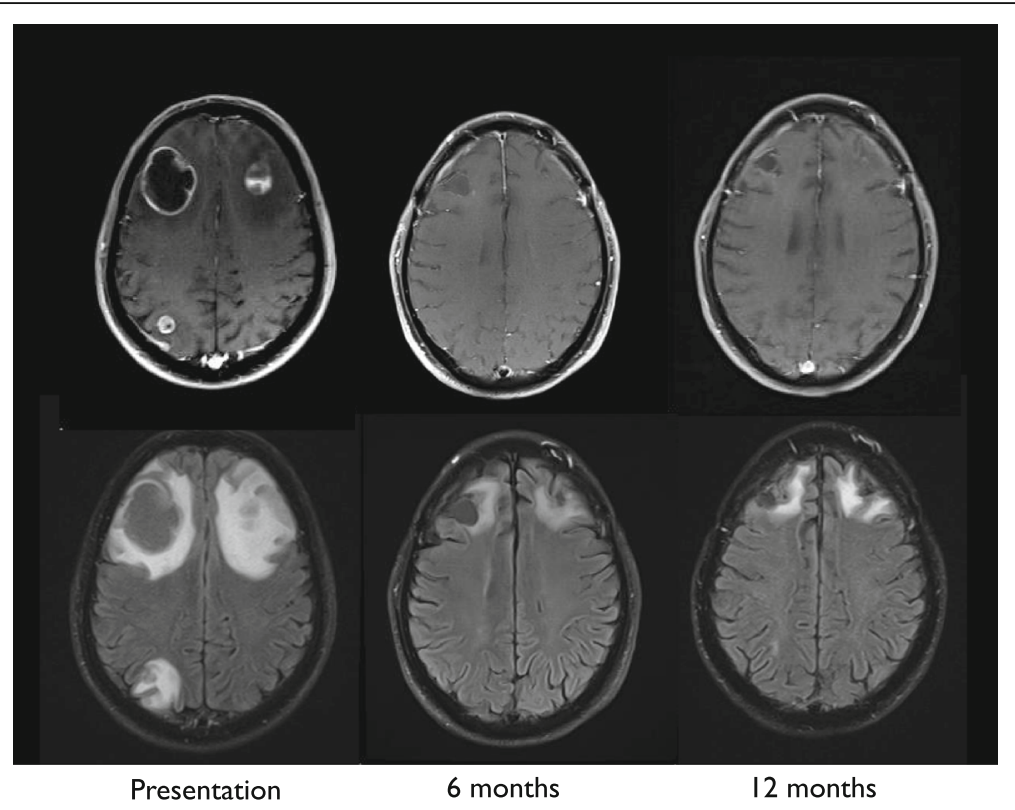

Fig. 1 Magnetic Resonance Imaging (MRI) brain: T1-weighted images following intravenous gadolinium-based contrast (top panel) and axial FLAIR images without contrast (bottom panel). MRI brain images taken at initial presentation show two large frontal lobe enhancing lesions at the gray-white matter junction with significant surrounding edema and associated T2 FLAIR hyperintensity. Post-treatment changes remain evident at 6 and 12 month follow-up scans 


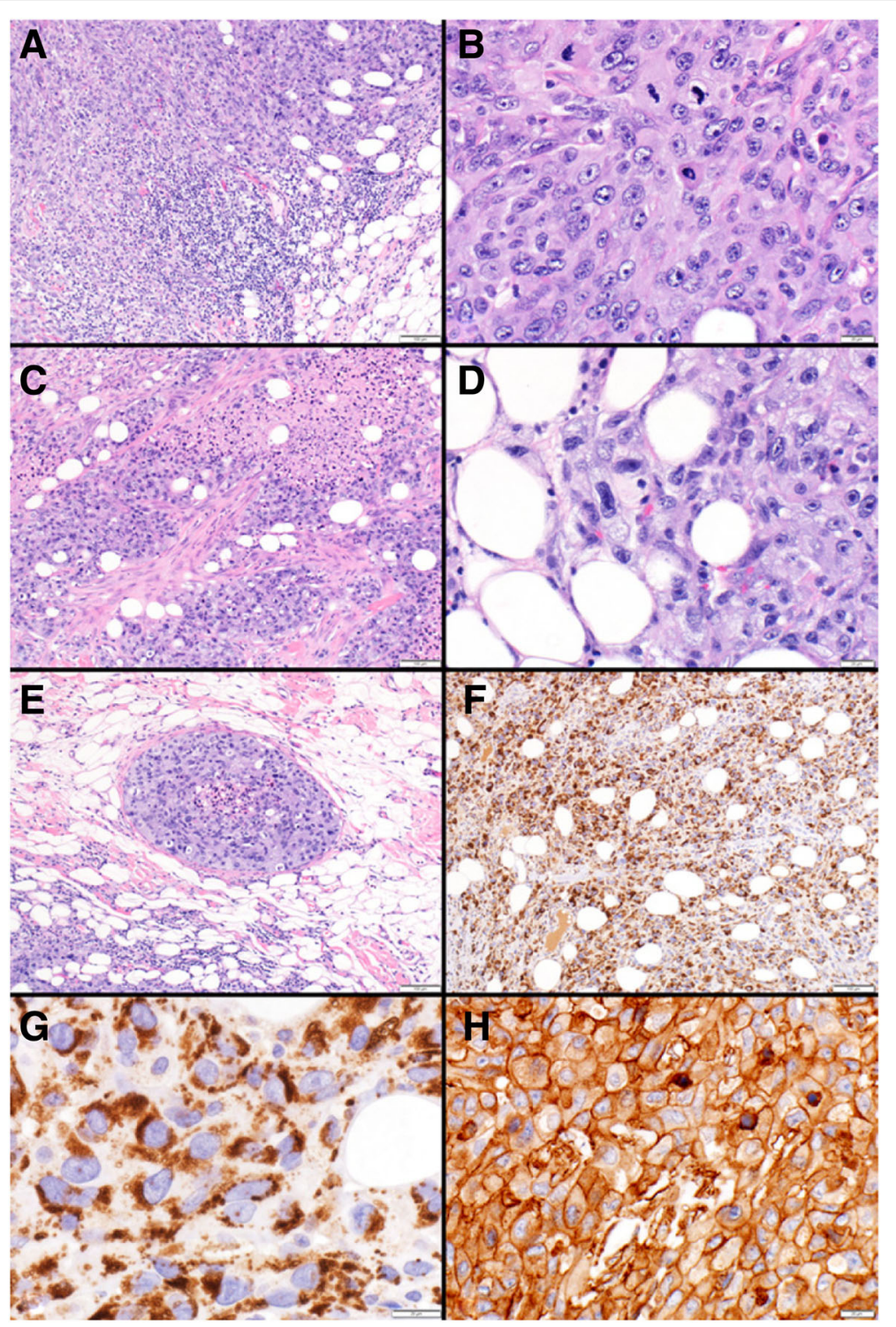

Fig. 2 Sebaceous carcinoma: Sheets of malignant cells are shown invading subcutaneous adipose tissue along with tumor infiltrating lymphocytes (lower right) (a). Malignant epithelial cells with nuclear pleomorphism, coarse chromatin, prominent nucleoli and increased mitotic activity (b). Carcinoma with stromal desmoplasia and tumor necrosis (c). Intracytoplasmic vacuolations suggest sebaceous differentiation (d). Carcinoma exhibiting lymphovascular space invasion (e). Adipophilin immunohistochemistry demonstrating diffuse (f) and characteristic strong membranous staining of the intracytoplasmic lipid vacuoles (g). PD-L1 (22C3, pharm Dx) immunohistochemistry showing positive (high, tumor proportion score > 50\%) expression in 100\% of tumor cells (h)

of life concerns. Given the strong rationale for use of checkpoint inhibitors in several other tumor types, moderately high tumor mutational burden (17 muts/Mb), and strong PD-L1 expression the patient opted for anti-PD1 immunotherapy. He initiated off-label treatment with pembrolizumab (2 $\mathrm{mg} / \mathrm{kg}$, every 3 weeks) in December 2016.

Follow up PET/ CT scan 3 months after the initiation of anti-PD1 therapy revealed remarkable improvement in lymph nodes, lung, and soft tissue, however, there were multiple new and enlarging hepatic and osseous metastases initially worrisome for progression(Fig. 3b). After multidisciplinary review, the findings were felt consistent with pseudo-progression and immunotherapy was continued. Restaging PET/ CT obtained after 6 months of treatment showed further significant improvements in all previously noted lesions (Fig. 3c), with residual FDG activity seen in small mediastinal and abdominal lymph nodes. To further characterize the patient's innate and adaptive immune status at the time of his near complete response, a flow cytometry study of peripheral blood was performed. Lymphocyte subset analysis showed evidence of 
Table 1 Tumor genomic next generation sequencing (NGS) study reveals mutations in several genes, including somatic mutations in genes mutated in the sebaceous carcinoma COSMIC dataset (in bold)

\begin{tabular}{|c|c|c|c|}
\hline Gene & Alteration & Subcellular localization & Pathway \\
\hline LRP1B & G3156C, Q1125* & Plasma membrane & Receptor mediated endocytosis \\
\hline CCND1 & S41L & Nucleus; cytosol & Cyclin D1, cell cycle \\
\hline TET2 & $\mathrm{P} 174 \mathrm{H}$ & Nucleoplasm & DNA demethylation \\
\hline TP53 & rearrangement, del exon 10-11 & Nucleoplasm & DNA repair \\
\hline FANCA & A816V, R685S & Nucleus & DNA repair \\
\hline FGF6 & A63T & Extracellular & Growth factor \\
\hline MYST3 & Q1681_Q1684del & Nucleolus; cytosol & Histone acetyltransferase (HAT) \\
\hline KRAS & G12C & Cytosol & MAPK signalling \\
\hline RBM10 & $\mathrm{F} 173 \mathrm{fs}^{* 7}$ & Nucleus & mRNA splicing \\
\hline MET & amplification & Plasma membrane & Receptor tyrosine kinase \\
\hline FGFR3 & 1539del & Endoplasmic reticulum & Receptor tyrosine kinase \\
\hline FLT4 & K520E, R658W & Nucleus; plasma membrane & Receptor tyrosine kinase \\
\hline ROS1 & $\mathrm{N} 692 \mathrm{H}$ & Vesicles & Receptor tyrosine kinase \\
\hline TERT & $-124 C>T$ & Nucleoplasm & Telomerase \\
\hline WT1 & R471S & Nucleoplasm & WT1 Transcription Factor \\
\hline MYC & L435F & Nucleoplasm & MYC Transcription Factor \\
\hline ZNF703 & G406R & Nucleus & Transcriptional co-repressor \\
\hline c11orf30 & C1211S & Nucleoplasm & Trascriptional Repressor \\
\hline FLCN & R320Q & Nucleus; cytosol & Tumor suppressor \\
\hline TNFAIP3 & R706Q & Cytosol & Ubiquitination \\
\hline
\end{tabular}

*denotes mutations causing a premature stop codon

fs denotes the presence of a frameshift mutation

circulating CD45RA-CD27+ central memory (CM) and effector memory (EM) T cells, and a population of mature CD16 + CD57+ NK cells (Fig. 4).

$\mathrm{He}$ continued on pembrolizumab, however, after 10 months of therapy he developed severe fatigue and orthostatic hypotension requiring hospitalization. Laboratory testing showed him to have secondary adrenal insufficiency with low $(<0.7 \mathrm{mcg} / \mathrm{dL})$ serum cortisol and low $(<11 \mathrm{pg} / \mathrm{mL})$ ACTH levels. He began $1 \mathrm{mg} / \mathrm{kg}$ prednisone with a prolonged steroid taper, and during high dose steroid therapy pembrolizumab dosing was interrupted. In December 2017, with 12 months of follow-up, restaging PET/CT was obtained and showed new FDG avid mediastinal and abdominal lymph nodes and a new hepatic and small bowel lesion (Fig. 3d). Biopsy of the liver lesion in January 2018 confirmed recurrent metastatic sebaceous carcinoma, and repeat immunohistochemical staining showed tumor cells continued to express high levels of PD-L1 (not shown). After discussion, the patient elected to restart immunotherapy, and he was continued on maintenance adrenal replacement therapy with hydrocortisone $(10 \mathrm{mg} / 5 \mathrm{mg})$. Recent restaging studies demonstrate growth of the mediastinal metastases and a reduction in the size of the hepatic and small bowel metastases, suggestive of pseudoprogression and a durable ongoing response to pembrolizumab (Additional file 1).

\section{Discussion and conclusions}

This is the first report of immune checkpoint inhibition in a patient with widely disseminated sebaceous carcinoma and brain metastases. Our patient was initially treated with brain-directed therapy and he recovered normal neurologic function. Systemic immunotherapy with anti-PD1 blockade led to a dramatic near complete response following 6 months of therapy. Initial tumor samples demonstrated strong and uniform PD-L1 expression on $100 \%$ of tumor cells (Fig. 1h), and the biopsy confirmed hepatic recurrence similarly expressed high levels of PD-L1. The ongoing response to pembrolizumab supports PD-L1 expression may be the most important biomarker predicting benefit from anti-PD1 therapy in sebaceous carcinoma. PD-L1 expression has been associated with response to pembrolizumab in advanced cutaneous melanoma [17], basal cell carcinoma [18], merkel cell carcinoma [19], and other tumors [20-22].

Several therapeutic targets derived from next generation sequencing were identified that could inform personalized and combinatorial treatment strategies. For 

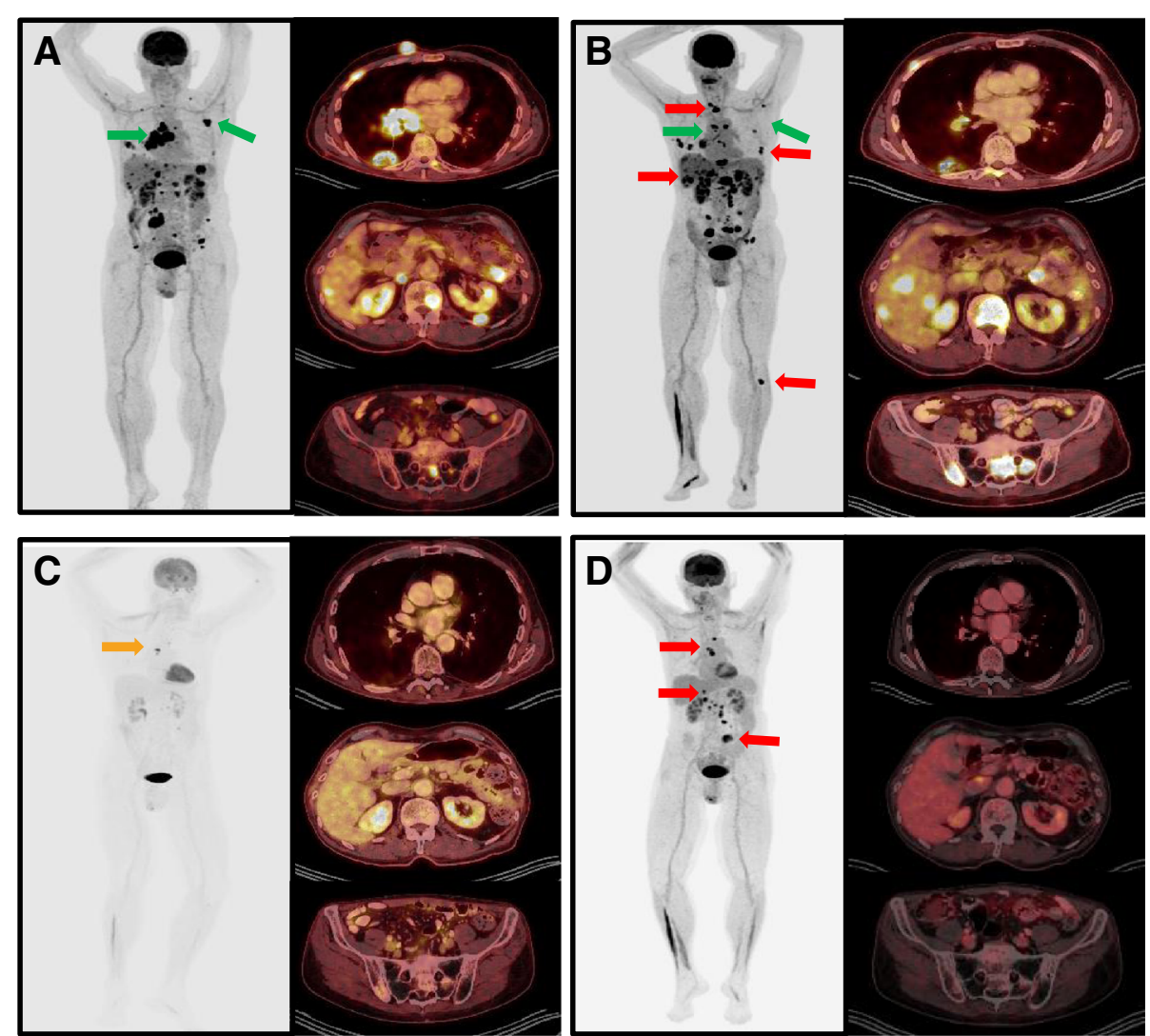

Fig. 3 Positron emission tomography (PET) maximum intensity projection (MIP) images and axial images from the chest, abdomen, and pelvis demonstrating the radiographic response to pembrolizumab therapy. a Baseline imaging shows extensive metastatic disease burden, and green arrows highlight lesions first to improve in lung, mediastinum, and axillary lymph nodes. b Imaging after 3 months (4 cycles) of pembrolizumab are consistent with mixed response and pseudoprogression, with several new hypermetabolic foci (red arrows) in soft tissue, liver and bone. $\mathbf{c}$ Imaging studies at 6 months shows a near complete response to 7 cycles of pembrolizumab, with regression of multiple hypermetabolic metastatic foci, and few small remaining foci of residual hypermetabolism in the chest and abdomen. $\mathbf{d}$ Imaging at 12 months shows persistent FDG avidity in mediastinal and abdominal lymph nodes and new FDG avid lesions in liver and small bowel (red arrows)

example, it is worthwhile to note amplification of the MET gene, whose involvement in tumor progression and metastasis in several cancers has been well documented [23-28]. This finding may be relevant in terms of potential combination therapy, with the ultimate goal of accomplishing a durable, synergistic therapeutic response. The combination of pembrolizumab and the c-Met inhibitor crizotinib is currently in phase 2 testing for Alk-positive non-small cell lung cancer (NCT02511184). The alterations in FGFR and FLT4 are similarly attractive targets for combination therapy in view of the antiangiogenic effects of their inhibition [29, 30].

The immunologic phenotyping study performed at 6 months (24 weeks) of therapy demonstrated that our patient's clinical response was associated with circulating CD8 + cytotoxic T lymphocytes in peripheral blood. The cytotoxic CD8 T cells had both central memory and effector memory phenotypes (Fig. 4c), as has been reported following vaccination against yellow fever and small pox [31].
Ribas and colleagues have also previously shown that tumor infiltrating CD8 memory $\mathrm{T}$ cells are associated with the response to anti-PD-1 therapy [32]. In anti-PD1 responders, memory CD8 $\mathrm{T}$ cells increased within the tumor microenvironment compared with non-responders after an average of 10 weeks; range 15-230 days [32]. While we did not specifically assess tumor infiltrating lymphocytes, our patient's clinical response suggests cytotoxic memory $\mathrm{T}$ cells may persist in the peripheral blood circulation. We also saw smaller populations of circulating effector T cells, similar to the report by Kamphorst and colleagues in non-small cell lung cancer patients responding to anti-PD1 immunotherapy [33]. Peripheral blood collected within 4 to 24 weeks of treatment in that study, showed increases in Ki-67 + CD8+ T cells lacking CD45RA and CCR7, consistent with $\mathrm{T}$ effector cell phenotype. Importantly, Ki-67 + CD8 + T cells also expressed high levels of PD-1 and CTLA-4, and CD28 and CD27 costimulatory molecules [33]. 

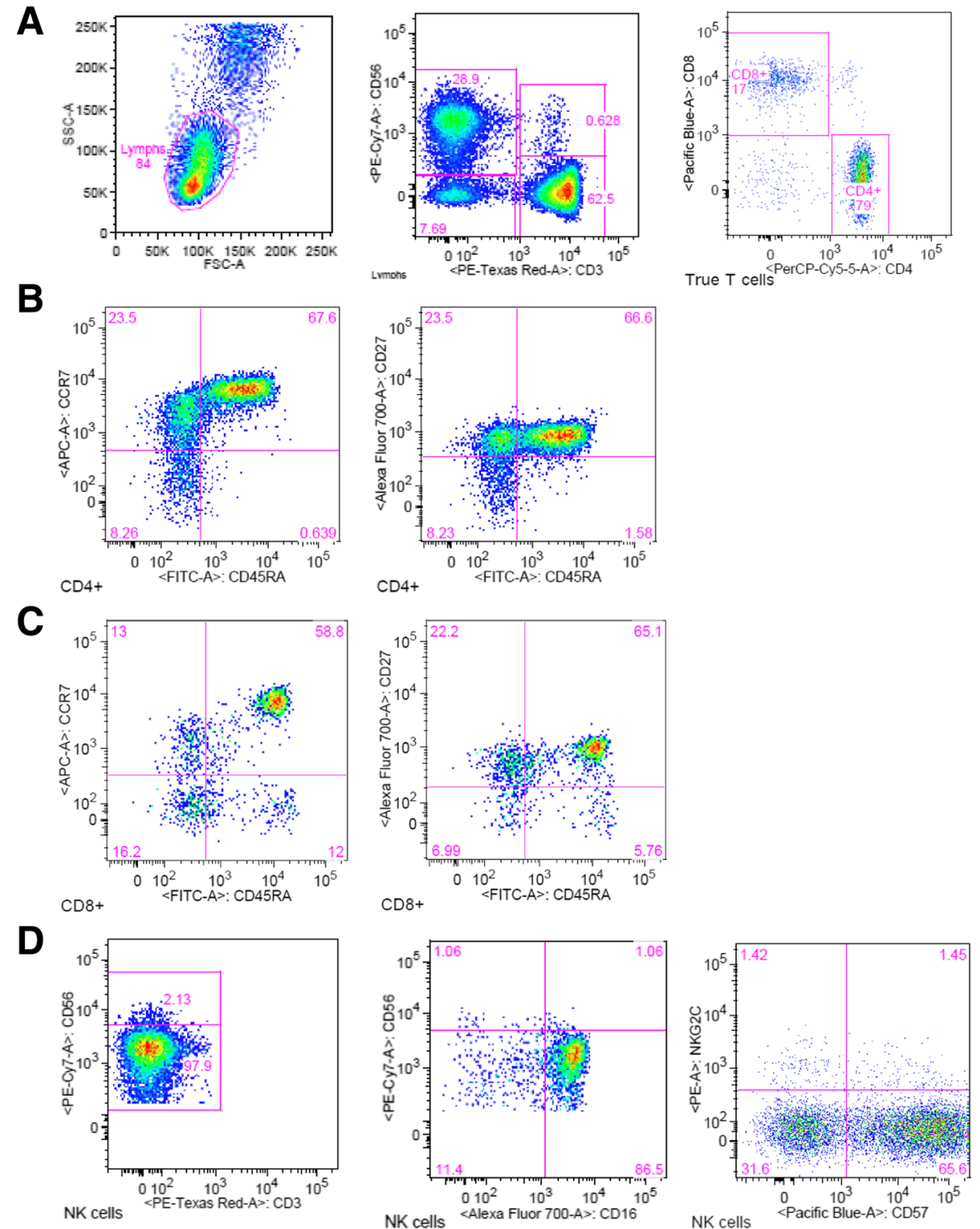

Fig. 4 Peripheral blood mononuclear cell (PBMC) phenotyping at 6-month (24 week) follow-up visit. a Dot plots show lymphocyte gating and the relative frequency of CD3-CD56+ NK cells and CD3+ T cells. b Dot plots show relative frequency of CD4 + CD45RA- memory T cells expressing CCR7 and CD27, and CD127 $7^{\circ}$ CD25+ regulatory T cells. $\mathbf{c}$ Dot plots of CD3 + CD8+ gated cells show that CD45RA-CD8+ T cells stain for memory markers CCR7 and CD27, and there are smaller populations of CD45RA + CCR7- and CD45RA + CD27- effector T cells. $\mathbf{d}$ NK cells are mature (CD56 dim), terminally differentiated (CD57+) and express the Fc-gamma receptor, CD16. Numbers inside dot plots indicate the percentage of cells for the markers analyzed

We hypothesize that chronic antigen stimulation by sebaceous carcinoma can stimulate adaptive immunity and $\mathrm{T}$ cell exhaustion, enabling the use of anti-PD1 checkpoint inhibition strategies. These preliminary results appear promising for this tumor histology, however, prospective clinical trials are needed. Early phase testing in the cooperative groups and clinical trial networks may enable more rapid translation of these findings to the clinic and support the tumor immunology research and centralized testing of clinical trial samples. Further detailed tumor antigen discovery and immune profiling during treatment of additional patients would allow for a clearer understanding of the adaptive immune response against both MSS and MSI-high sebaceous carcinoma tumors.

In conclusion, the ongoing, durable response to checkpoint inhibition described in this report supports clinical testing of anti-PD1 checkpoint inhibitors in MSS and MSI-high sebaceous carcinoma. Prospective, open-label 
clinical testing is warranted to further define the role of front-line immunotherapy for the treatment of advanced, metastatic sebaceous carcinoma.

\section{Additional file}

Additional file 1 : Figure S1. Coronal and axial contrast enhanced CTimages were obtained at 13 months and shows mediastinal and liver recurrence. Repeat imaging at 15 months shows increased size of the mediastinal lesion (top row), and decreased size of the hepatic lesion (bottom row), suggesting mixed immune related responses and pseudoprogression. (DOCX $600 \mathrm{~kb}$ )

\section{Acknowledgements}

Authors wish to thank Martin Felices, PhD and Julie Curtsinger, PhD for assistance with flow cytometric testing and review of the final manuscript.

\section{Authors' contributions}

All authors contributed to the care of the patient and were involved in the generation of figures and writing of the manuscript. All authors read and approved the final manuscript.

\section{Ethics approval and consent to participate}

Patient described in this case signed a case report informed consent form which is available for review.

\section{Consent for publication}

Patient consented to publication of the case report

\section{Competing interests}

The authors declare that they have no competing interests.

\section{Publisher's Note}

Springer Nature remains neutral with regard to jurisdictional claims in published maps and institutional affiliations.

\section{Author details}

${ }^{1}$ Department of Medicine, Division of Hematology, Oncology and Transplantation, University of Minnesota, 420 Delaware Street SE, MMC480, Minneapolis, MN 55455, USA. ${ }^{2}$ Department of Laboratory Medicine and Pathology, University of Minnesota, Minneapolis, MN 55455, USA. ${ }^{3}$ Department of Radiology, University of Minnesota, Minneapolis, MN 55455, USA. ${ }^{4}$ Department of Radiation Oncology, University of Minnesota, Minneapolis, MN 55455, USA. ${ }^{5}$ Department of Neurosurgery, University of Minnesota, Minneapolis, MN 55455, USA. ${ }^{6}$ Masonic Cancer Center, University of Minnesota, Minneapolis, MN 55455, USA

\section{Received: 2 January 2018 Accepted: 15 May 2018}

Published online: 19 June 2018

\section{References}

1. Deprez M, Uffer S. Clinicopathological features of eyelid skin tumors. A retrospective study of 5504 cases and review of literature. Am J Dermatopathol. 2009;31(3):256-62.

2. Bailet JW, et al. Sebaceous carcinoma of the head and neck. Case report and literature review. Arch Otolaryngol Head Neck Surg. 1992;118(11):1245-9.

3. Nelson BR, et al. Sebaceous carcinoma. J Am Acad Dermatol. 1995;33(1):115. quiz $16-8$

4. Tripathi $\mathrm{R}$, et al. Incidence and survival of sebaceous carcinoma in the United States. J Am Acad Dermatol. 2016:75(6):1210-5.

5. Dasgupta T, Wilson LD, Yu JB. A retrospective review of 1349 cases of sebaceous carcinoma. Cancer. 2009;115(1):158-65.

6. Orcurto A, et al. Long-term remission of an aggressive sebaceous carcinoma following chemotherapy. Case Rep Dermatol. 2014;6(1):80-4.

7. Kumar $V, X u Y$. Unusual presentation of metastatic sebaceous carcinoma and its response to chemotherapy: is genotyping a right answer for guiding chemotherapy in rare tumours? Curr Oncol. 2015;22(4):e316-9.
8. Gaskin BJ, et al. The significance of DNA mismatch repair genes in the diagnosis and management of periocular sebaceous cell carcinoma and Muir-Torre syndrome. Br J Ophthalmol. 2011;95(12):1686-90.

9. Cortes-Ciriano I, et al. A molecular portrait of microsatellite instability across multiple cancers. Nat Commun. 2017:8:15180.

10. Schachter J, et al. Pembrolizumab versus ipilimumab for advanced melanoma: final overall survival results of a multicentre, randomised, openlabel phase 3 study (KEYNOTE-006). Lancet. 2017;390(10105):1853-62.

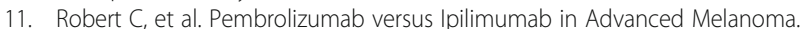
N Engl J Med. 2015;372(26):2521-32.

12. Nghiem PT, et al. PD-1 blockade with Pembrolizumab in advanced Merkelcell carcinoma. N Engl J Med. 2016;374(26):2542-52.

13. Kaufman $\mathrm{HL}$, et al. Avelumab in patients with chemotherapy-refractory metastatic Merkel cell carcinoma: a multicentre, single-group, open-label, phase 2 trial. Lancet Oncol. 2016:17(10):1374-85.

14. Boussahmain C, Mochel MC, Hoang MP. Perilipin and adipophilin expression in sebaceous carcinoma and mimics. Hum Pathol. 2013;44(9):1811-6.

15. Jakobiec FA, Mendoza PR. Eyelid sebaceous carcinoma: clinicopathologic and multiparametric immunohistochemical analysis that includes adipophilin. Am J Ophthalmol. 2014;157(1):186-208. e2

16. Forbes SA, et al. COSMIC: somatic cancer genetics at high-resolution. Nucleic Acids Res. 2017:45(D1):D777-83.

17. Long GV, et al. PD-L1 expression as a biomarker for nivolumab (NIVO) plus ipilimumab (IPI) and NIVO alone in advanced melanoma (MEL): a pooled analysis. Ann Oncol. 2016;27

18. Lipson EJ, et al. Basal cell carcinoma: PD-L1/PD-1 checkpoint expression and tumor regression after PD-1 blockade. J Immunother Cancer. 2017:5:23.

19. Lipson EJ, et al. PD-L1 expression in the Merkel cell carcinoma microenvironment: association with inflammation, Merkel cell polyomavirus and overall survival. Cancer Immunol Res. 2013;1(1):54-63.

20. Aguiar PN Jr, et al. The role of PD-L1 expression as a predictive biomarker in advanced non-small-cell lung cancer: a network meta-analysis. Immunotherapy. 2016;8(4):479-88.

21. Khunger M, et al. Meta-analysis of tumor PD-L1 expression as a predictive biomarker of benefit from PD-1/PD-L1 axis inhibitors in solid tumors. J Clin Oncol. 2016;34(15)

22. Wang $X$, et al. PD-L1 expression in human cancers and its association with clinical outcomes. Onco Targets Ther. 2016;9:5023-39.

23. Bradley CA, et al. Targeting c-MET in gastrointestinal tumours: rationale, opportunities and challenges. Nat Rev Clin Oncol. 2017;14(9):562-76.

24. Straussman $\mathrm{R}$, et al. Tumour micro-environment elicits innate resistance to RAF inhibitors through HGF secretion. Nature. 2012;487(7408):500-4.

25. Tu WH, et al. Efficacy of c-Met inhibitor for advanced prostate cancer. BMC Cancer. 2010;10:556.

26. Chia SK, et al. A phase-I study of lapatinib in combination with foretinib, a c-MET, AXL and vascular endothelial growth factor receptor inhibitor, in human epidermal growth factor receptor 2 (HER-2)-positive metastatic breast cancer. Breast Cancer Res. 2017:19(1):54

27. Eder JP, et al. A phase I study of foretinib, a multi-targeted inhibitor of cMet and vascular endothelial growth factor receptor 2. Clin Cancer Res. 2010;16(13):3507-16

28. Leon LG, et al. Synergistic activity of the c-Met and tubulin inhibitor tivantinib (ARQ197) with pemetrexed in mesothelioma cells. Curr Drug Targets. 2014;15(14):1331-40.

29. Lieu C, et al. Beyond VEGF: inhibition of the fibroblast growth factor pathway and antiangiogenesis. Clin Cancer Res. 2011;17(19):6130-9.

30. Langenberg $\mathrm{MH}$, et al. Phase I evaluation of telatinib, a vascular endothelial growth factor receptor tyrosine kinase inhibitor, in combination with irinotecan and capecitabine in patients with advanced solid tumors. Clin Cancer Res. 2010;16(7):2187-97.

31. Miller JD, et al. Human effector and memory CD8+ T cell responses to smallpox and yellow fever vaccines. Immunity. 2008;28(5):710-22.

32. Ribas A, et al. PD-1 blockade expands Intratumoral memory T cells. Cancer Immunol Res. 2016:4(3):194-203.

33. Kamphorst AO, et al. Proliferation of PD-1+CD8 T cells in peripheral blood after PD-1-targeted therapy in lung cancer patients. Proc Natl Acad Sci U S A. 2017;114(19):4993-8. 